\title{
INFLUÊNCIA DE M/C (M = Mo, Cu, Fe E W) INCORPORADO À CAMADA DIFUSORA DO ELETRODO DE DIFUSÃO DE GÁS FRENTE À REAÇÃo DE OXIDAÇÃO DE HIDROGÊNIO NA PRESENÇA DE CO
}

\author{
Luis Gustavo da Silva Pereira, Maristela Elisabete Pereira e Edson Antonio Ticianelli* \\ Departamento de Físico-Química, Instituto de Química de São Carlos, Universidade de São Paulo, CP 780, 13560-970 São
}

Carlos - SP, Brasil

Recebido em 25/9/06; aceito em 14/2/07; publicado na web 30/7/07

\begin{abstract}
THE EFFECT OF M/C ( $\mathrm{M}=\mathrm{Mo}, \mathrm{Cu}, \mathrm{Fe}$ AND W) IN THE DIFFUSION LAYER OF GAS DIFFUSION ELECTRODES ON THE HYDROGEN OXIDATION REACTION IN THE PRESENCE OF CARBON MONOXIDE. The performance of proton exchange membrane fuel cells (PEMFC) with Pt-based anodes is drastically lowered when CO-containing hydrogen is used to feed the system, because of the strong adsorption of $\mathrm{CO}$ on platinum. In the present work the effects of the presence of a conversion layer of $\mathrm{CO}$ to $\mathrm{CO}_{2}$ composed by several $\mathrm{M} / \mathrm{C}$ materials (where $\mathrm{M}=\mathrm{Mo}, \mathrm{Cu}, \mathrm{Fe}$ and $\mathrm{W}$ ) in gas diffusion anodes formed by Pt catalysts were investigated. The diffusion layers formed by Mo/C e W/C show good CO-tolerance, and this was attributed to the $\mathrm{CO}$ removal by parallel occurrence of the water-gas shift reaction and the so-called bifunctional mechanism.
\end{abstract}

Keywords: PEMFC; carbon monoxide; water-gas shift.

\section{INTRODUÇÃO}

A busca por fontes alternativas de energia é uma tendência mundial e, neste contexto, as células a combustível constituem a mais promissora alternativa para a geração de energia elétrica em substituição aos geradores acionados por motores a combustão. $\mathrm{O}$ combustível ideal para as células a combustível com membrana trocadora de prótons (PEMFC - "proton exchange membrane fuel cell") é o hidrogênio puro, mas seu uso é pouco favorecido por causa das dificuldades de armazenamento e manuseio. Mesmo na forma líquida ou ainda na forma de um hidreto metálico, há uma enorme preocupação e severas exigências de medidas de segurança. Uma alternativa a este problema seria que o hidrogênio utilizado na célula fosse produzido "a bordo" ou in loco através da reforma do gás natural, metanol ou etanol. No entanto, o processo de reforma, além de hidrogênio, produz também $\mathrm{CO}$, que se adsorve fortemente sobre a superfície do catalisador convencional de $\mathrm{Pt} / \mathrm{C}$ (nanopartículas de platina ancorada em carbono), afetando drasticamente o desempenho da célula, mesmo quando traços deste contaminante estão presentes ${ }^{1-3}$. Desta forma, a tolerância ao monóxido de carbono é um fator importante na escolha de um catalisador para a oxidação anódica de hidrogênio em células PEMFC alimentadas com o gás reformado.

Algumas abordagens significativas têm sido propostas para solucionar o problema de contaminação do eletrodo por CO. Uma destas abordagens está relacionada à presença de um segundo elemento além da $\mathrm{Pt}$, como $\mathrm{Ru}^{4,5}, \mathrm{Sn}^{6,7}, \mathrm{Mo}^{8,9}$ e Os ${ }^{10,11}$, formando ligas ou co-depósitos dispersos em carbono, resultando em um incremento significativo na tolerância ao $\mathrm{CO}$ quando comparado ao catalisador que é formado por partículas de platina pura. Vários tipos de mecanismos têm sido adotados para explicar a maior eficiência da eletrooxidação de $\mathrm{H}_{2}$ contaminado por $\mathrm{CO}$ sobre $\mathrm{Pt}-\mathrm{Ru}$, dentre os quais destacam-se o mecanismo bifuncional (mecanismo promovido) $)^{12}$ e o efeito eletrônico (mecanismo intrínseco) ${ }^{13}$. De acordo com o mecanismo bifuncional, a espécie reagente $\left(\mathrm{H}_{2}\right)$ e o contaminante $(\mathrm{CO})$ adsorvem-se preferencialmente nos átomos de

*e-mail: edsont@iqsc.usp.br
Pt (Equações 1-3), enquanto que o outro metal, menos nobre e conseqüentemente mais oxidável $(\mathrm{M}=\mathrm{Sn}, \mathrm{Ru}$ etc), produz espécies oxigenadas ou óxidos hidratados que atuam diretamente na oxidação do contaminante (Equações 4-5)

$$
\begin{aligned}
& \mathrm{H}_{2}+2 \mathrm{Pt} \rightleftharpoons 2 \mathrm{Pt}-\mathrm{H} \\
& \mathrm{Pt}-\mathrm{H} \rightleftharpoons \mathrm{Pt}+\mathrm{H}^{+}+\mathrm{e}^{-} \\
& \mathrm{CO}+\mathrm{Pt} \rightleftharpoons \mathrm{Pt}-\mathrm{CO} \\
& \mathrm{M}+\mathrm{H}_{2} \mathrm{O} \rightleftharpoons \mathrm{M}-\mathrm{OH}+\mathrm{H}^{+}+\mathrm{e}^{-} \\
& \mathrm{Pt}-\mathrm{CO}+\mathrm{M}-\mathrm{OH} \rightleftharpoons \mathrm{Pt}+\mathrm{M}+\mathrm{CO}_{2}+\mathrm{H}^{+}+\mathrm{e}^{-}
\end{aligned}
$$

O mecanismo intrínseco postula que a presença do segundo metal modifica a energia de quimissorção de $\mathrm{H}_{2}$ e $\mathrm{CO}$ por um mecanismo de doação/retrodoação de elétrons que enfraquecem a ligação Pt-CO.

Além da formação de liga, outra abordagem empregada para reduzir os efeitos do envenenamento por $\mathrm{CO}$ consiste na aplicação de pulsos de corrente na célula durante sua operação com CO. De maneira simplificada, aumentando a densidade de corrente da célula durante a aplicação do pulso há uma redução da voltagem da célula. Como consequiência do pulso, o material anódico é forçado a operar transientemente em valores mais positivos de potencial, suficientes para proceder à oxidação eletroquímica de $\mathrm{CO}$ a $\mathrm{CO}_{2}{ }^{14}$ Outra abordagem envolve a inserção de uma pequena quantidade de ar (1 a 6\% em volume) juntamente com o fluxo de combustível, sendo dessa maneira o $\mathrm{CO}$ adsorvido sobre a Pt cataliticamente oxidado a $\mathrm{CO}_{2}{ }^{15}$. Trabalhos recentes relatam que o uso de óxidos de metais de transição suportado em carbono proporciona uma oxidação mais efetiva do $\mathrm{CO}$ a $\mathrm{CO}_{2}$ quando na presença de $\operatorname{ar}^{16,17}$.

Recentemente, Ticianelli e colaboradores propuseram a aplicação de eletrodos de difusão de gás contendo uma pré-camada de conversão química de $\mathrm{CO}$ em $\mathrm{CO}_{2}$ baseada em $\mathrm{Mo} / \mathrm{C}^{18,19}$ como forma de aumentar a tolerância ao envenenador da reação. Para este sistema os autores observaram um aumento significativo na tolerância ao $\mathrm{CO}$ em eletrocatalisadores de Pt/C (perda de potencial de $140 \mathrm{mV}$ a $1 \mathrm{~A} \mathrm{~cm}^{-2}$ ) e atribuíram este efeito à diminuição da concentração de $\mathrm{CO}$ nos canais gasosos do eletrodo de difusão de gás, causada pela reação de deslocamento do equilíbrio gáságua (WGS - "water gas shift") representada por 


$$
\mathrm{CO}+\mathrm{H}_{2} \mathrm{O} \rightleftharpoons \mathrm{CO}_{2}+\mathrm{H}_{2}
$$

Apesar dos avanços na área de células a combustível de membrana de troca protônica, o desenvolvimento de novos materiais eletrocatalisadores e sistemas eletródicos mais tolerantes ao $\mathrm{CO}$ ainda é imperioso. Assim, neste trabalho foi feito um estudo sistemático do desempenho de eletrodos de difusão de gás com Pt/C na camada catalisadora e contendo potenciais agentes conversores de $\mathrm{CO}$ em $\mathrm{CO}_{2}$ formados por catalisadores dispersos em carbono baseados em Mo e W, que apresentam uma elevada atividade eletrocatalítica para a reação de oxidação de hidrogênio na presença de $\mathrm{CO}$ e, também, em $\mathrm{Fe}$ e $\mathrm{Cu}$, que em catálise heterogênea são conhecidos catalisadores da reação de $\mathrm{WGS}^{20}$.

\section{PARTE EXPERIMENTAL}

Os agentes conversores de $\mathrm{CO}$ compostos por $\mathrm{M} / \mathrm{C}$ (onde $\mathrm{M}=$ $\mathrm{Mo}, \mathrm{Cu}, \mathrm{Fe}$ e W) foram preparados utilizando-se método de impregnação/decomposição ${ }^{21}$. Este consiste na dissolução do sal do metal em estudo em álcool etílico, com adição posterior de pó de carbono (Vulcan - XC 72, Cabot); em seguida, todo o sistema é mantido em ultra-som por $3 \mathrm{~h}$, com evaporação posterior do solvente, sendo o material resultante tratado a $100{ }^{\circ} \mathrm{C}$ em atmosfera de argônio por $1 \mathrm{~h}$ e a $550{ }^{\circ} \mathrm{C}$ em atmosfera de hidrogênio por $3 \mathrm{~h}$. Na Tabela 1 estão listados os reagentes utilizados no preparo dos agentes conversores, bem como seu respectivo fabricante e especificação.

Tabela 1. Relação dos reagentes utilizados na preparação dos agentes conversores

\begin{tabular}{lccc}
\hline $\begin{array}{l}\text { Agente } \\
\text { conversor }\end{array}$ & Reagente & Fabricante & Especificação \\
\hline $\mathrm{Mo} / \mathrm{C}$ & $\left(\mathrm{NH}_{4}\right)_{6} \mathrm{Mo}_{7} \mathrm{O}_{24} \cdot 4 \mathrm{H}_{2} \mathrm{O}$ & Mallinckrodt & P.A \\
$\mathrm{Cu} / \mathrm{C}$ & $\mathrm{CuSO}_{4} \cdot 5 \mathrm{H}_{2} \mathrm{O}$ & Merck & $99 \%$ \\
$\mathrm{Fe} / \mathrm{C}$ & $\mathrm{FeSO}_{4}$ & Carlo Erba & $89 \%$ \\
$\mathrm{~W} / \mathrm{C}$ & $\mathrm{Na}_{2} \mathrm{WO}_{4} \cdot 2 \mathrm{H}_{2} \mathrm{O}$ & Riedel - deHaën & $99,8 \%$ \\
\hline
\end{tabular}

Os materiais produzidos foram caracterizados fisicamente através de análises de difração de raios $\mathrm{X}(\mathrm{DRX})$ e energia dispersiva de raios $\mathrm{X}(\mathrm{EDX})$. Os difratogramas de DRX foram obtidos com um difratômetro URD-6 Carl Zeiss-Jena a uma velocidade de varredura de $2^{\circ} \mathrm{min}^{-1}$ e com intervalo de $2 \theta$ entre $20^{\circ}$ e $60^{\circ}$. O comprimento de onda da radiação incidente foi de $1,5406 \AA$, que corresponde à banda $\mathrm{CuK} \alpha$. Para as análises de EDX fez-se uso de um espectrômetro Zeiss-Leica/440 com detector de SiLi.

No presente trabalho, além da configuração tradicional dos eletrodos de difusão de gás (EDG), usou-se uma configuração alternativa para este eletrodo. Na Figura 1 tem-se uma representação esquemática dos dois sistemas, sendo que em ambos a camada catalítica do ânodo é constituída por Pt/C (E-Tek) e Nafion ${ }^{\circledR}$. A diferença fundamental destas configurações reside no fato de que no sistema tradicional a camada difusora é formada somente por pó de carbono (Vulcan - XC 72, Cabot), tecido de carbono (PWB3, Stackpole), suspensão de politetrafluoretileno (PTFE - Teflon T30 , DuPont) e solução de Nafion (Aldrich, 5 wt.\%) ${ }^{22}$. Na configuração alternativa, além destes materiais, tem-se a camada difusora formada por metais não nobres dispersos em carbono (agentes conversores de $\mathrm{CO}$ em $\mathrm{CO}_{2}$ ).

O conjunto membrana-eletrodos (MEA - "membrane electrode assembly") foi preparado por prensagem a quente na temperatura de $125^{\circ} \mathrm{C}$ e $1000 \mathrm{kgf} \mathrm{cm}^{-2}$ durante $2 \mathrm{~min}$. Em todos os casos foram utilizadas membranas de Nafion 115 (forma $\mathrm{H}^{+}$, DuPont) como eletrólito. $\mathrm{O}$ sistema membrana-eletrodos foi acondicionado entre

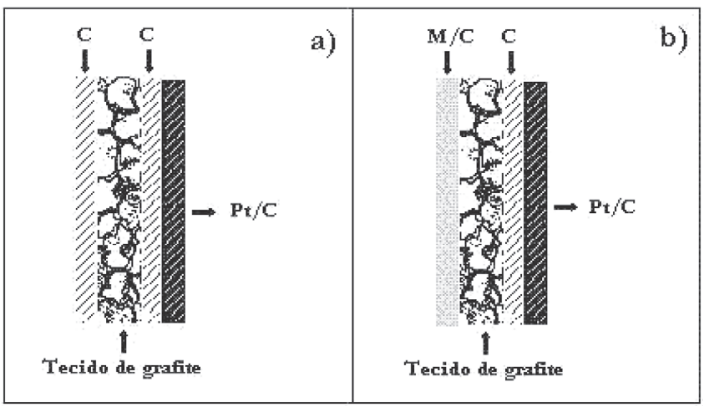

Figura 1. Representação do EDG: a) em sua configuração convencional e b) em sua configuração alternativa. $M=\mathrm{Mo}, \mathrm{Cu}, \mathrm{Fe} e \mathrm{~W} ; \mathrm{C}=$ pó de carbono Vulcan XC- 72 com PTFE

placas de grafite que permitem o contato elétrico e a distribuição uniforme do fluxo dos gases que alimentam a célula. $O$ conjunto todo foi acondicionado entre placas terminais de alumínio, sendo que através destas ocorrem a coleta da eletricidade e o controle de aquecimento da célula unitária.

Curvas de polarização em estado estacionário em células a combustível unitárias foram obtidas galvanostaticamente, mantendose a célula a $85^{\circ} \mathrm{C}$ e utilizando-se oxigênio saturado com água ultrapura (>18 M, Milli-Q) a $90{ }^{\circ} \mathrm{C}$ e pressão de 1,7 atm. O desempenho eletrocatalítico dos ânodos foi avaliado utilizando-se hidrogênio puro ou $\mathrm{H}_{2} / \mathrm{CO}$ (100 ppm) saturados com água ultrapura a $100{ }^{\circ} \mathrm{C}$ e $2 \mathrm{~atm}$. Anteriormente às medidas, o sistema foi acondicionado em $0,7 \mathrm{~V} / 2 \mathrm{~h}$ para as medidas com hidrogênio puro e em 0,8 $\mathrm{V} / 2 \mathrm{~h}$ para a mistura $\mathrm{H}_{2} / \mathrm{CO}(100 \mathrm{ppm})$ com o objetivo de se alcançar a condição de estado estacionário.

As análises por voltametria cíclica foram realizadas apenas nos ânodos sendo que ao cátodo baseado em $\mathrm{Pt} / \mathrm{C}$, que atuou como eletrodo de referência $\left(\mathrm{Pt}, \mathrm{H}_{2} / \mathrm{H}^{+} \mathrm{ERH}\right)$ e como contra-eletrodo, foi injetado o gás $\mathrm{H}_{2}$. Ao ânodo (eletrodo de trabalho) foi injetado nitrogênio constantemente para desoxigenação do sistema. Os voltamogramas cíclicos foram realizados em um potenciostatogalvanostato (Solarton 1285) com intervalo de potencial entre 0,1 e 1,0 V versus ERH (eletrodo reversível de hidrogênio) a uma velocidade de varredura de $10 \mathrm{mVs}^{-1}$.

Medidas de espectrometria diferencial de massas (DEMS"differential electrochemical mass spectrometry") foram realizadas visando um melhor entendimento do processo de oxidação de CO que ocorre nos ânodos e, para isso, um espectrômetro de massa (Omnistar) foi acoplado à saída do ânodo da célula unitária para medidas "on line" da quantidade de $\mathrm{CO}_{2}$ formado.

\section{RESULTADOS E DISCUSSÃO}

Na Tabela 2 estão reunidos os resultados das análises de EDX dos agentes conversores em estudo. Pode-se verificar que o método empregado (impregnação/decomposição) foi altamente eficiente, uma vez que a composição obtida para os materiais se encontra próxima à composição nominal, exceção feita ao material que contém ferro. Uma possível explicação para o desvio da composição obtida em relação à nominal para o material baseado em $\mathrm{Fe} / \mathrm{C}$ pode estar relacionada à presença de impurezas no precursor utilizado $\left(\mathrm{FeSO}_{4}\right)$, tendo em vista que o mesmo apresenta um grau de pureza da ordem de $90 \%$.

Os padrões de difração de raios X dos agentes conversores M/ C estão apresentados na Figura 2. Através de pesquisa junto às fichas cristalográficas do JCPDS ("The Joint Committee for Powder Diffraction Studies"), identificou-se que o material baseado em $\mathrm{Fe} / \mathrm{C}$ apresenta reflexões características de $\mathrm{Fe}_{2} \mathrm{O}_{3}$ (óxido de ferro) 
Tabela 2. Porcentagem em massa dos agentes conversores obtida por EDX

\begin{tabular}{lcccc}
\hline $\begin{array}{l}\text { Agente } \\
\text { conversor }\end{array}$ & \multicolumn{2}{c}{$\begin{array}{c}\text { Composição } \\
\text { nominal }(\% \text { massa) } \\
\text { M }\end{array}$} & C & \multicolumn{2}{c}{$\begin{array}{c}\text { Composição } \\
\text { obtida } \\
\text { (\% massa) }\end{array}$} \\
\hline $\mathrm{Mo} / \mathrm{C}$ & 20 & 80 & 19 & $\mathbf{\text { M }}$ \\
$\mathrm{Cu} / \mathrm{C}$ & 20 & 80 & 16 & 84 \\
$\mathrm{Fe} / \mathrm{C}$ & 20 & 80 & 9 & 91 \\
$\mathrm{~W} / \mathrm{C}$ & 20 & 80 & 30 & 70 \\
\hline
\end{tabular}

e $\mathrm{FeC}$ (carbeto de ferro). Para o agente conversor que contém Mo em sua composição $(\mathrm{Mo} / \mathrm{C})$ apenas reflexões características de $\mathrm{MoO}_{2}$ foram verificadas. Analisando o material baseado em $\mathrm{Cu} / \mathrm{C}$ observa-se a presença de picos de difração de $\mathrm{CuO}$ e $\mathrm{Cu}$. Tais resultados indicam que as condições experimentais (atmosfera de $\mathrm{H}_{2}$ e temperatura de $550{ }^{\circ} \mathrm{C}$ ) do método proposto geram materiais com estrutura baseada em $\mathrm{MO}_{x}$. Entretanto, para o agente conversor baseado em W/C o tratamento térmico em atmosfera de $\mathrm{H}_{2}$ não se mostrou eficaz para a redução do tungstênio, uma vez que as reflexões observadas para este material estão relacionadas às do agente precursor utilizado $\left(\mathrm{Na}_{2} \mathrm{WO}_{4}\right)$.

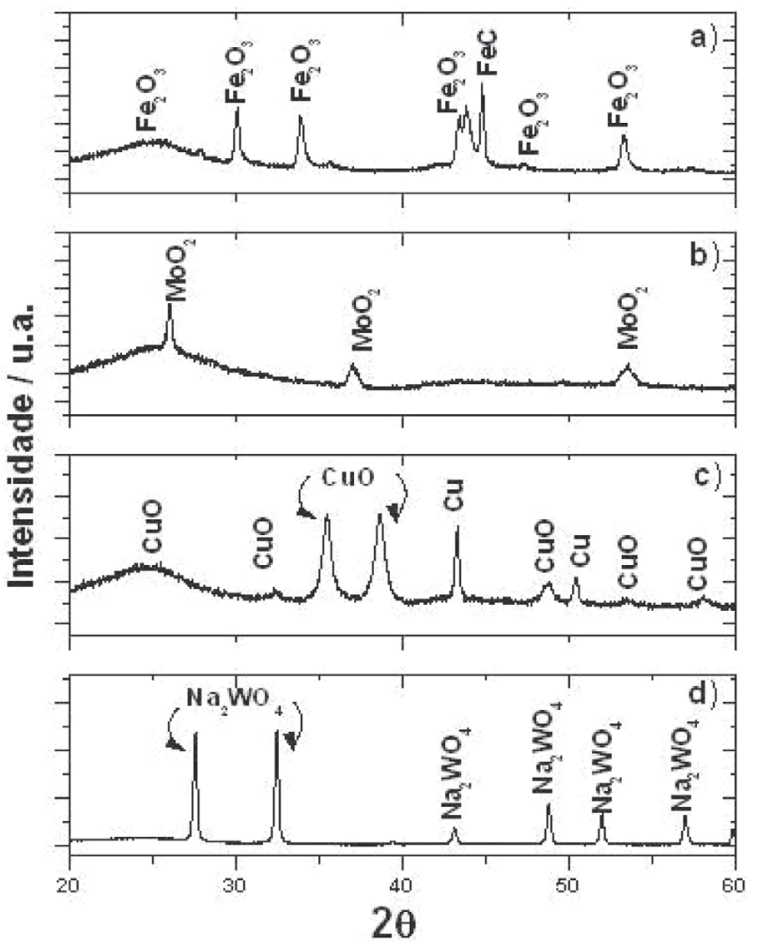

Figura 2. Padrões de difração de raios $X$ dos agentes conversores sintetizados por impregnação/decomposição, onde (a) $\mathrm{Fe} / \mathrm{C}$, (b) $\mathrm{Mo} / \mathrm{C}$, (c) $\mathrm{Cu} / \mathrm{C}$ e (d) W/C

As curvas de polarização para sistemas eletródicos contendo camada conversora de $\mathrm{CO}$ à base de $\mathrm{Fe} / \mathrm{C}, \mathrm{Cu} / \mathrm{C}, \mathrm{Mo} / \mathrm{C}$ e W/C incorporados na camada difusora (Figura 1) estão apresentadas nas Figuras 3 a 6. Para efeito de comparação, em todas as figuras foi apresentada a resposta típica do eletrodo com a camada difusora convencional formada somente com carbono.

Analisando-se os resultados das Figuras 3 e 4 nota-se claramente que para os ânodos com os materiais à base de $\mathrm{Fe}$ e $\mathrm{Cu}$ há uma perda da atividade catalítica em relação ao eletrodo convencional de $\mathrm{Pt} / \mathrm{C}$ (E-Tek) mesmo na presença de $\mathrm{H}_{2}$ puro. Isto indica que a presença dos agentes conversores em questão $(\mathrm{Fe} / \mathrm{C}$ e $\mathrm{Cu} / \mathrm{C})$ afe-

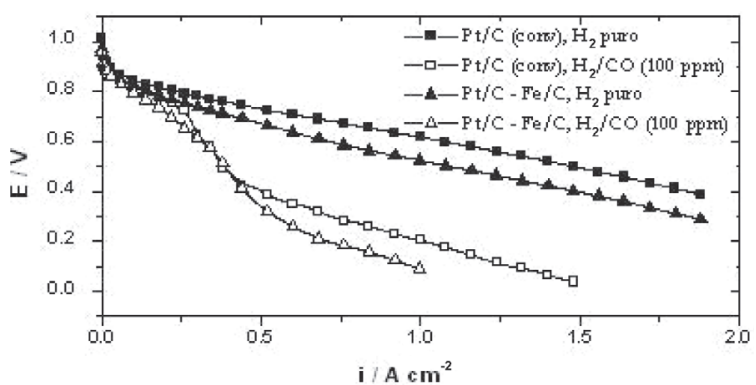

Figura 3. Curvas de polarização em estado estacionário de células a combustível unitárias utilizando catalisadores anódicos de Pt/C (camada difusora convencionall) e Pt/C - Fe/C (camada difusora contendo agente conversor de $\mathrm{Fe} / \mathrm{C}$ ) alimentados com $\mathrm{O}_{2}$ no cátodo e $\mathrm{H}_{2}$ e $\mathrm{H}_{2} / \mathrm{CO}$ (100 ppm) no ânodo. $T_{\text {célula }}=85^{\circ} \mathrm{C}$

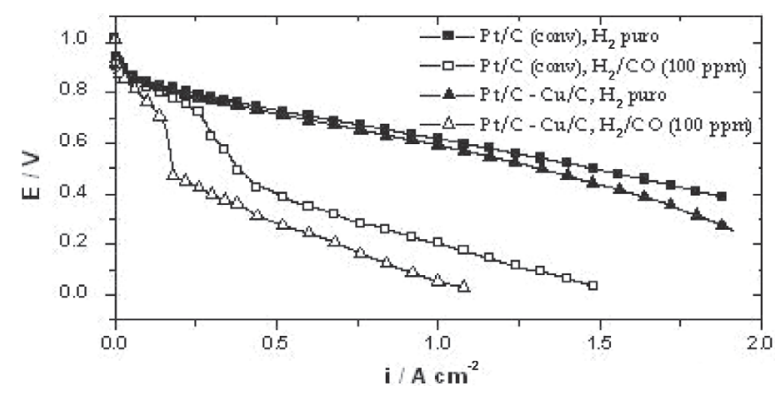

Figura 4. Curvas de polarização em estado estacionário de células a combustível unitárias utilizando catalisadores anódicos de Pt/C (camada difusora convencional) e Pt/C - Cu/C (camada difusora contendo agente conversor de $\mathrm{Cu} / \mathrm{C}$ ) alimentados com $\mathrm{O}_{2}$ no cátodo e $\mathrm{H}_{2}$ e $\mathrm{H}_{2} / \mathrm{CO}$ (100 ppm) no ânodo. $T_{\text {célula }}=85^{\circ} \mathrm{C}$

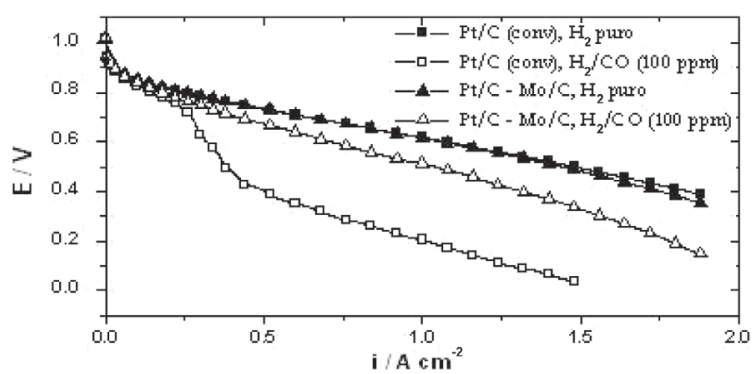

Figura 5. Curvas de polarização em estado estacionário de células a combustivel unitárias utilizando catalisadores anódicos de Pt/C (camada difusora convencional) e Pt/C - Mo/C (camada difusora contendo agente conversor de $\mathrm{Mo} / \mathrm{C}$ ) alimentados com $\mathrm{O}_{2}$ no cátodo e $\mathrm{H}_{2}$ e $\mathrm{H}_{2} / \mathrm{CO}$ (100 ppm) no ânodo. $T_{\text {célula }}=85^{\circ} \mathrm{C}$

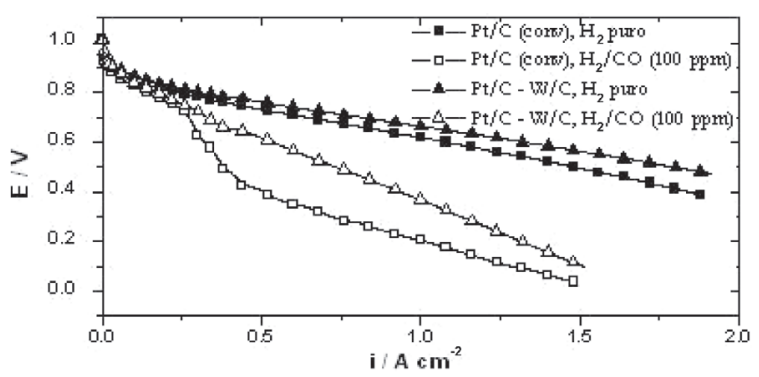

Figura 6. Curvas de polarização em estado estacionário de células a combustível unitárias utilizando catalisadores anódicos de Pt/C (camada difusora convencional) e Pt/C - W/C (camada difusora contendo agente conversor de W/C) alimentados com $\mathrm{O}_{2}$ no cátodo e $\mathrm{H}_{2}$ e $\mathrm{H}_{2} / \mathrm{CO}$ (100 ppm) no ânodo. $T_{\text {célula }}=85^{\circ} \mathrm{C}$ 
ta de maneira negativa a reação de oxidação de hidrogênio em relação ao eletrodo convencional de Pt com carbono na camada difusora. Os resultados também mostram que na presença de $\mathrm{H}_{2} / \mathrm{CO}$ (100 ppm) não há incremento da tolerância ao $\mathrm{CO}$, ou seja, não se observa a ocorrência do mecanismo representado na reação WGS (Equação 6) ${ }^{18,19}$ para os eletrodos com a camada conversora baseada em $\mathrm{Fe}$ e $\mathrm{Cu}$. Estes materiais são mencionados como bons promotores desta reação ${ }^{20}$, mas isto se verifica em temperaturas consideravelmente maiores que aquelas utilizadas nas condições normais de operação da célula PEMFC $\left(85^{\circ} \mathrm{C}\right.$, no presente caso).

Por outro lado, os resultados para o eletrodo contendo $\mathrm{Mo} / \mathrm{C}$ (Figura 5) indicam um desempenho frente a $\mathrm{ROH}$ na ausência de $\mathrm{CO}$ similar ao do eletrodo convencional. Também, um aumento da tolerância ao CO é observado para o eletrodo em sua configuração alternativa. Finalmente, observa-se que o material contendo camada conversora à base de W/C (Figura 6) resultou em comportamento muito semelhante ao do $\mathrm{Mo} / \mathrm{C}$. Comparado à Pt pura, nota-se um aumento da tolerância ao $\mathrm{CO}$ para o catalisador quando na presença da camada de W/C, apesar de o efeito ser inferior ao do Mo/C.

Para os materiais que apresentaram boa tolerância em relação ao $\mathrm{CO}$ foram realizadas medidas de voltametria cíclica e de espectrometria diferencial de massas (DEMS) no intuito de verificar o mecanismo operacional do processo. Estes resultados são apresentados, respectivamente, nas Figuras 7 e 8.

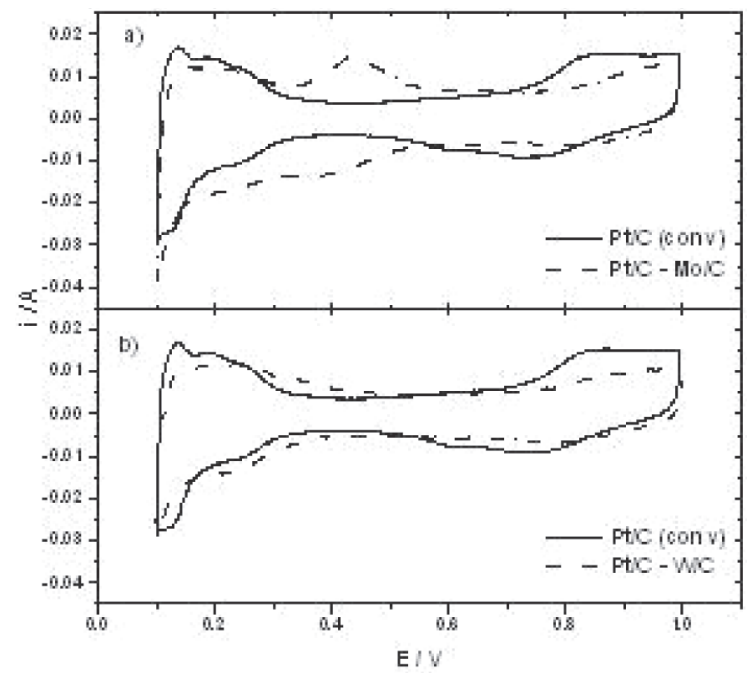

Figura 7. a) Voltamogramas cíclicos à temperatura ambiente para células unitárias operando com eletrodos de difusão de gás obtidos a partir de catalisador comercial (Pt/C, E-Tek) e com a camada difusora contendo agente conversor de $\mathrm{Mo/C}$; b) voltamogramas cíclicos à temperatura ambiente para células unitárias operando com eletrodos de difusão de gás obtidos a partir de catalisador comercial (Pt/C, E-Tek) e com a camada difusora contendo agente conversor de W/C. $T_{\text {célula }}=85^{\circ} \mathrm{C}$

Na Figura 7a são mostrados os voltamogramas cíclicos para os eletrodos que contêm a camada conversora baseada em Mo/C. A resposta para o eletrodo convencional com $\mathrm{Pt} / \mathrm{C}$ é aquela tipicamente encontrada para este material. Para o eletrodo com $\mathrm{Mo} / \mathrm{C}$ na camada difusora verifica-se que há um pico bem definido em torno de $0,5 \mathrm{~V}$ que tem sido atribuído por alguns autores ao processo redox $\mathrm{Mo}(\mathrm{IV}) \rightarrow \mathrm{Mo}(\mathrm{VI})$ ou $\mathrm{Mo}(\mathrm{V}) \rightarrow \mathrm{Mo}(\mathrm{VI})^{19,24}$, evidenciando uma pequena interpenetração das camadas difusora e catalisadora. $\mathrm{Na}$ Figura $7 \mathrm{~b}$ têm-se os voltamogramas cíclicos para os eletrodos com filtros de W/C. Observa-se que estes eletrodos apresentam um perfil voltamétrico semelhante ao que contém filtros de $\mathrm{Mo} / \mathrm{C}$, destacando-se, em particular, a região de adsorção/dessorção de

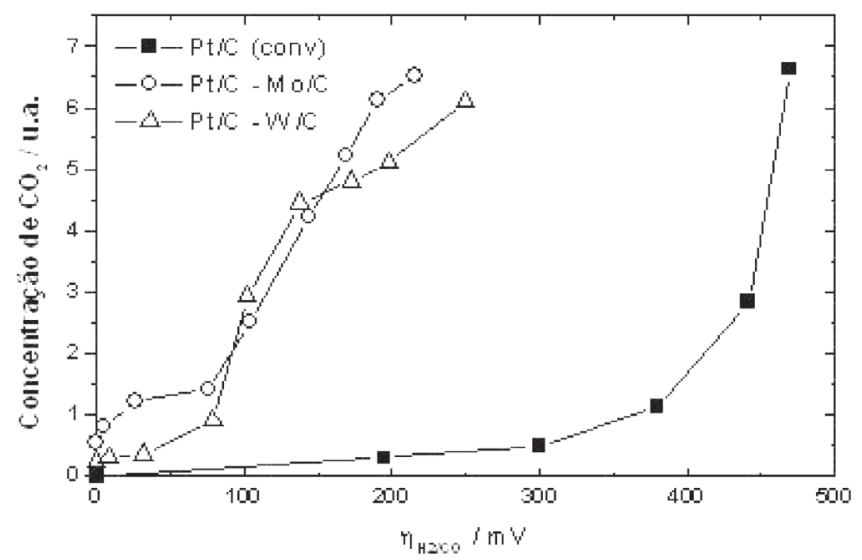

Figure 8. Produção de $\mathrm{CO}_{2}$ versus sobrepotencial do eletrodo de hidrogênio obtida por DEMS para Pt/C (camada difusora convencional), $\mathrm{Pt} / \mathrm{C}-\mathrm{Mo} / \mathrm{C}$ (camada difusora contendo agente conversor de Mo/C) e Pt/C - W/C (camada difusora contendo agente conversor de $\mathrm{W} / \mathrm{C}) . T_{\text {célula }}=85{ }^{\circ} \mathrm{C}$

hidrogênio está relativamente bem definida e o surgimento de um pico em torno de $0,35 \mathrm{~V}$ relacionado a algum processo do $\mathrm{WO}_{4}^{-2} 25$. Assim, verifica-se que em ambos os casos há um claro processo de interpenetração entre a camada conversora de $\mathrm{CO}(\mathrm{Mo} / \mathrm{C}$ e W/C) e a camada catalítica $(\mathrm{Pt} / \mathrm{C})$ ocorrida, provavelmente, durante a etapa de prensagem. Neste caso, algumas partículas de Mo e W podem ter entrado em contato com o eletrólito impregnado na camada catalisadora, sofrendo assim os processos de oxi-redução verificados na Figura 7.

A produção de $\mathrm{CO}_{2}$ foi monitorada em função do sobrepotencial do eletrodo de hidrogênio $\left(\eta_{\mathrm{H} 2 / \mathrm{CO}}\right)$, definido como sendo a diferença entre o potencial da célula na ausência e na presença de $\mathrm{CO}$, por medidas on line de DEMS para a célula unitária a $85^{\circ} \mathrm{C}$, como mostra a Figura 8. Para ambos os sistemas (Pt/C - Mo/C e Pt/C - W/C) verifica-se que a produção de $\mathrm{CO}_{2}$ é maior em uma ampla faixa de potenciais, além de que se inicia em potenciais inferiores quando comparado ao sistema tradicional sem a camada conversora de CO.

Tais resultados vêm corroborar a idéia da ocorrência de uma etapa química (WGS, Equação 6) na remoção do contaminante da reação, uma vez que mesmo em circuito aberto $\left(\eta_{\mathrm{H} / \mathrm{CO}}=0\right)$ pôde-se verificar a formação de $\mathrm{CO}_{2}$. No entanto, a ocorrência do mecanismo bifuncional (Equações 1-5) é comprovada pela acentuada variação da taxa da produção de $\mathrm{CO}_{2}$ com o potencial da célula, conforme previsto pelas reações 4 e 5 . Este último processo constitui o principal mecanismo de remoção do $\mathrm{CO}$, sendo favorecido pela presença de espécies de Mo e W que entram em contato íntimo com a Pt da camada catalisadora do eletrodo, conforme evidenciado pelos voltamogramas cíclicos apresentados na Figura 7.

\section{CONCLUSÕES}

A tolerância frente ao $\mathrm{CO}$ para os eletrodos confeccionados a partir das camadas difusoras baseadas em Mo/C e W/C dá-se pela ocorrência paralela de uma etapa eletroquímica (mecanismo bifuncional) e uma etapa química (WGS) de conversão de $\mathrm{CO}$ a $\mathrm{CO}_{2}$. $\mathrm{O}$ mesmo não é válido para os materiais à base de $\mathrm{Fe}$ e $\mathrm{Cu}$, que na temperatura de operação da célula não favoreceram a ocorrência de qualquer reação química ou eletroquímica de oxidação de CO. Destaca-se que as medidas de DEMS feitas por acoplamento do espectrômetro na saída do ânodo da célula PEMFC é pioneira, não havendo sido ainda, no melhor do nosso conhecimento, mencionada na literatura. Estas medidas foram essenciais na identificação do mecanismo de tolerância ao $\mathrm{CO}$, sendo que o fato mais 
contundente foi a observação da produção de $\mathrm{CO}_{2}$ em circuito aberto, que corrobora de forma inequívoca a idéia da ocorrência de uma etapa química de oxidação de CO.

\section{AGRADECIMENTOS}

Ao Conselho Nacional de Desenvolvimento Cientifico e Tecnológico (CNPq) e à Fundação de Amparo à Pesquisa do Estado de São Paulo (FAPESP) pelo suporte financeiro.

\section{REFERÊNCIAS}

1. Steele, B. C. H.; Heinzel, A.; Nature 2001, 414, 345.

2. Murthy, M.; Esayian, M.; Hobson, A.; Mackenzie, S.; Lee, W.; van Zee, J. W.; J. Electrochem. Soc. 2001, 148, A1141.

3. Chalk, S. G.; Miller, J. F.; Wagner, F. W.; J. Power Sources 2000, 86, 40.

4. Gu, T.; van Zee, J. W.; Murthy, M.; J. Electrochem. Soc. 2004, 151, A2100.

5. Ralph, T. R.; Hogarth, M. P.; Platinum Metals Rev. 2002, 46, 117.

6. Zhou, W. J.; Song, S. Q.; Li, W. Z.; Zhou, Z. H.; Sun, G. Q.; Xin, Q.; Douvartzides, S.; Tsiakaras, P.; J. Power Sources 2005, 140, 50.

7. Camara, G. A.; Ticianelli, E. A.; Mukerjee, S.; Lee, S. J.; McBreen, J.; J. Electrochem. Soc. 2002, 149, A748.

8. Götz, M.; Wendt, H.; Electrochim. Acta 1998, 43, 3637.

9. Mukerjee, S.; Urian, R. C.; Lee, S. J.; Ticianelli, E. A.; McBreen, J.; J. Electrochem. Soc. 2004, 151, A1094.

10. Santiago, E. I.; Ticianelli, E. A.; Int. J. Hydrogen Energy 2005, 30, 159
11. Santiago, E. I.; Giz, M. J.; Ticianelli, E. A.; J. Solid State Electrochem. 2003, 7, 607 .

12. Watanabe, M.; Motoo, S.; J. Electroanal. Chem. 1975, 60, 267.

13. Christoffersen, E.; Liu, P.; Ruban, A.; Skriver, H. L.; Norskov, J. K.; J. Catal. 2001, 199, 123

14. Carrette, L. P. L.; Friedrich, K. A.; Huber, M.; Stimming, U.; Phys. Chem. Chem. Phys. 2001, 3, 320.

15. Gottesfeld, S.; Pafford, J.; J. Electrochem. Soc. 1998, 135, 2651.

16. Adcock, P. A.; Pacheco, S. V.; Norman, K. M.; Uribe, F. A.; J. Electrochem. Soc. 2005, 152, A459.

17. Uribe, F. A.; Valerio, J. A.; Garzon, F. H.; Zawodzinski, T. A.; Electrochem. Solid-State Lett. 2004, 7, A376.

18. Santiago, E. I.; Batista, M. S.; Assaf, E. M.; Ticianelli, E. A.; J. Electrochem. Soc. 2004, 151, A944.

19. Santiago, E. I.; Camara, G. A.; Ticianelli, E. A.; Electrochim. Acta 2003 48,3527

20. Tanaka, Y.; Kikuchi, R.; Sasaki, K.; Eguchi, K.; Appl. Catal. 2003, $238,11$.

21. Santiago, E. I.; Paganin, V. A.; Carmo, M.; Gonzalez, E. R.; Ticianelli, E. A.; J. Electroanal. Chem. 2005, 575, 53.

22. Paganin, V. A.; Ticianelli, E. A.; Gonzalez, E. R.; J. Appl. Electrochem. 1996, 26, 297.

23. Pereira, L. G. S.; Santos, F. R.; Pereira, M. E.; Paganin, V. A.; Ticianelli, E. A.; Electrochim. Acta 2006, 51, 4061.

24. Li, W. S.; Tian, L. P.; Huang, Q. M.; Li, H.; Chen, H. Y.; Lian, X. P.; J. Power Sources 2002, 104, 281.

25. Machida, K.; Enyo, M.; Adachi, G.; Shiokawa, J.; J. Electrochem. Soc. 1988, 135, 1955. 